\title{
Reducing Fear of Reinjury and Pain Perception in Athletes With First-Time Anterior Cruciate Ligament Reconstructions by Implementing Imagery Training
}

\author{
Rosa M. Rodriguez, Ashley Marroquin, and Nicole Cosby
}

\begin{abstract}
Clinical Scenario: The anterior cruciate ligament is one of the major stabilizing ligaments of the knee joint by preventing anterior translation of the femur in the closed kinetic chain. A ruptured anterior cruciate ligament may require reconstructive surgery for patients who wish to return to physical activity. For the most part, surgeries are successful at repairing the ruptured ligament and restoring ligamentous function; the percentage of athletes that return to a competitive level of physical activity is only $44 \%$, and $24 \%$ of patients report a main factor of preventing their return is fear of reinjury and pain. Most physiotherapy and rehabilitation research has focused on the physical treatment and is limited on the psychological aspects of recovery. Imagery has been suggested to be effective at reducing anxiety, tension, and pain, while promoting and encouraging healing after an injury. Imagery is defined as a process of performing a skill in one's mind using the senses (touch, feel, smell, vision, etc) without any overt actions. Clinical Question: In athletes who are first-time anterior cruciate ligament reconstruction patients, does imagery training in combination with standard physical therapy reduce the fear of reinjury and pain perception? Summary of Key Findings: Previous research has primarily looked at the physical treatment aspect, and few studies have focused on the psychological factors affecting recovery. Researchers concluded that fear of reinjury was the unique predictor of return to sport even in a sample of participants that reported very little or almost no pain at all. Imagery as a therapy is an effective intervention in reducing fear of reinjury and confidence building. Furthermore, mental imagery is suggested to assist with a reduction in anxiety, pain, and tension, while promoting healing. Clinical Bottom Line: Based on the strength of recommendation taxonomy, there is a combination of level A and B evidence proposing that imagery, in combination with traditional physical therapy, can be effective at reducing psychological distress such as fear of reinjury and pain perception in first-time anterior cruciate ligament reconstruction patients.
\end{abstract}

Keywords: knee joint, psychology, rehabilitation, sport psychology

\section{Clinical Scenario}

Injuries are an inevitable part of competitive sports as well as recreational activities. Research shows that one of the most common musculoskeletal complaints that accounts for approximately 48 out of 1000 patients is knee-related injury. ${ }^{1}$ As noted, approximately 9\% of these knee complaints are related to anterior cruciate ligament (ACL) injuries. ${ }^{2,3}$ The ACL is commonly injured in contact sports by a noncontact action, such as planting, cutting, landing on a hyperextended knee, or pivoting and sudden deceleration. ${ }^{4}$ Typically, athletes report feeling immediate pain and hearing a pop, resulting in progressive knee swelling and difficulty ambulating. The ACL is one of the major ligaments that provides stability to the knee joint by preventing anterior translation of the femur in the closed kinetic chain. ${ }^{5}$ Therefore, most of the time, a ruptured ACL results in reconstructive surgery aimed at repairing the ligament. Athletes will often choose reconstructive surgery in an effort to return to physical activity. A major outcome measure that assesses the success of an ACL reconstructive surgery is the ability to return to sports or recreational activity. ${ }^{6}$ Although surgery is effective at repairing the ruptured ligament and restoring ligamentous function, for the most part, the percentage of athletes that return to a competitive level of physical activity is only $44 \% .^{4}$ Of these, $44 \%$ report not returning to preinjury activity and $24 \%$ report

The authors are with Point Loma Nazarene University, San Diego, CA. Rodriguez (Rmrodriguez711@pointloma.edu) is corresponding author. that the main factor preventing their return is fear of reinjury and pain. ${ }^{4,7}$ The inability to return to the previous level of activity can be stressful and mentally frustrating for athletes. Taking into consideration how the psychological aspects of the injury affect an athlete's sports performance, it is crucial to address psychological factors during physical rehabilitation for an athlete to rebuild confidence and overcome fear of reinjury. Currently, most physiotherapy and rehabilitation research has primarily focused on the physical treatment without focusing on the psychological aspects of recovery following an injury. ${ }^{5,7}$ The use of imagery as a psychological intervention has been suggested to be effective at reducing anxiety, tension, and pain, while promoting and encouraging healing after an injury. Imagery is defined as a process of performing a skill in one's mind using the senses (touch, feel, smell, vision, etc) without any overt actions. ${ }^{8}$ Incorporating a therapeutic intervention that targets the psychological effects on an athletes' ability to return to sport is necessary to assure that the patient is both physically and mentally prepared to return to activity. Therefore, the purpose of the critically appraised topic was to determine if imagery training in combination with standard physical therapy is a suitable option to reduce the fear of reinjury and pain perception among first-time anterior cruciate ligament reconstruction (ACLR) patients.

\section{Focused Clinical Question}

In athletes who are first-time ACLR patients, does imagery training in combination with standard physical therapy reduce fear of reinjury and pain perception? 


\section{Summary of Search, Best Evidence Appraised, and Key Findings}

- Previous research has primarily looked at the physical treatment aspect, and few studies have focused on the psychological factors affecting recovery. ${ }^{5}$

- Researchers have concluded that fear of reinjury was the unique predictor of return to sport even in a sample of participants that reported very little or almost no pain at all. ${ }^{9}$

- A critical appraisal of the literature revealed that imagery as a therapy is an effective intervention in reducing the fear of reinjury and confidence building. Furthermore, mental imagery is suggested to assist with a reduction in anxiety, pain, and tension, while promoting healing. ${ }^{7}$

\section{Clinical Bottom Line}

There is moderate to strong evidence indicating that many patients with ACLRs experience psychological factors that impede their ability to return to preinjury level of activity. Evidence also suggests that this patient population would benefit from interventions that target both the physical and psychological aspects of rehabilitation. These interventions may aid the efforts of reducing the percentage of patients with ACLRs that do not return to preinjury activity due to fear and pain perception.

\section{Strength of Recommendation}

Based on the strength of recommendation taxonomy, there is a combination of level A and B evidence proposing that psychological factors are expressed as an important aspect that affects rehabilitation and return to preinjury activity in this patient population during the course of 6- to 12 -months postoperative.

\section{Search Strategy}

\section{Terms Used to Guide Search Strategy}

- Patient group: ACLR patients

- Intervention: Imagery in combination with standard physical therapy

- Comparison: Only standard physical therapy

- Outcomes: Reduce fear of reinjury and pain perception when returning to preinjury level of activity

\section{Sources of Evidence Searched}

- PubMed

- EBSCOhost

- The Cochrane Library via EBSCOhost

- Google Scholar

\section{Inclusion and Exclusion Criteria (Include Search Limits)}

\section{Inclusion Criteria}

- Studies investigating ACL surgery outcomes

- Subjects with history of minimum of 1 surgery
Table 1 Summary of Study Designs of Articles Retrieved

\begin{tabular}{lcc}
\hline $\begin{array}{l}\text { Level of } \\
\text { evidence }\end{array}$ & $\begin{array}{c}\text { Study design/methodology } \\
\text { of articles retrieved }\end{array}$ & Author \\
\hline 1B & RCT & Maddison et al ${ }^{8}$ \\
1B & RCT & Cupal and Brewer \\
1A & RCT & Lebon et al ${ }^{10}$ \\
2B & RCT & Wilczynska et al $^{11}$ \\
\hline
\end{tabular}

Abbreviation: RCT, randomized controlled trials.

- Studies using imagery as an intervention

- Studies investigating psychological factors affecting rehabilitation

- Studies with at least 2B level of evidence and minimum of 5 on the PEDro scale

- Studies within the last 15 years

- Restricted to the English language

\section{Exclusion Criteria}

- Studies using subjects with a history of bilateral knee surgeries

- Studies that include participants with more than 1 reported ACLR

- Studies investigating other interventions other than a type of imagery as a form of intervention

\section{Results of Search}

Four relevant studies $5,8,10,11$ were located and categorized, as shown in Table 1 (based on Levels of Evidence, Centre for Evidence Based Medicine, 2011).

\section{Best Evidence}

The studies shown in Table 1 were identified as the best evidence. These studies were graded a level of evidence of $1 \mathrm{~A}, 1 \mathrm{~B}$, and 2B. They investigated imagery as an intervention to address physical and psychological factors affecting patients with ACLRs.

\section{Summary of Best Evidence}

Characteristics of included studies are shown in Table 2.

\section{Implications for Practice, Education, and Future Research}

Surgical reconstruction is aimed at restoring mechanical knee stability, which, in turn, is supposed to promote optimal function. It is noted that surgical recovery is mainly focused on improving motion and strength of the knee joint, while reducing psychological distress has not been stressed as an important aspect in postoperative ACLR rehabilitation. ${ }^{12}$ In efforts to address psychological factors affecting this population, the studies appraised have suggested that the fear of reinjury may play a significant role 


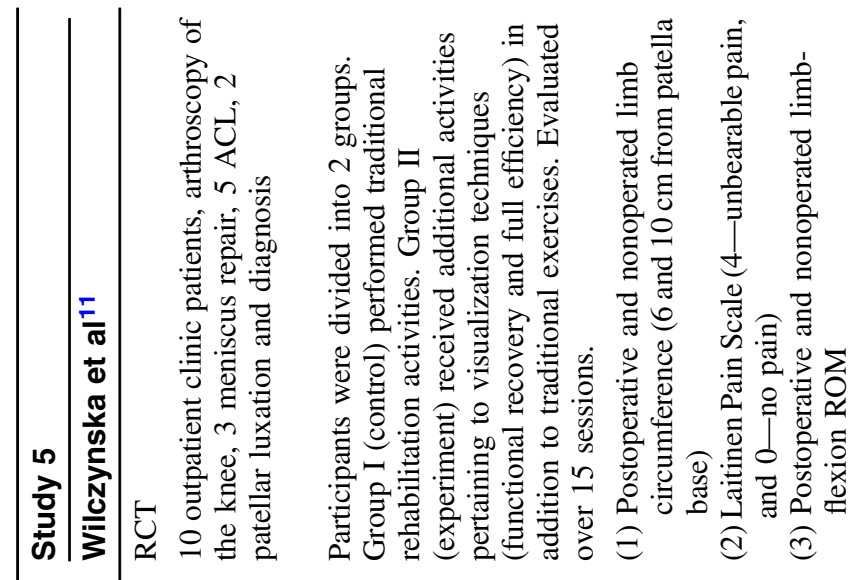

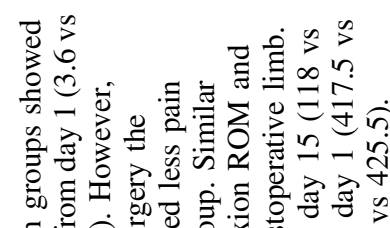

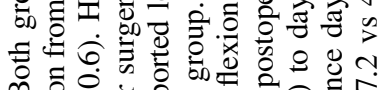

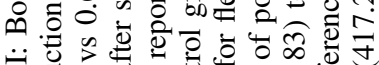



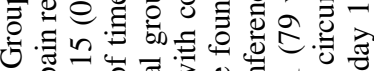

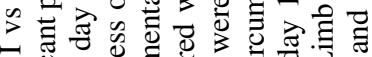

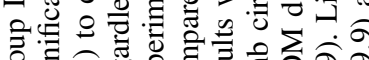

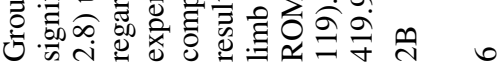
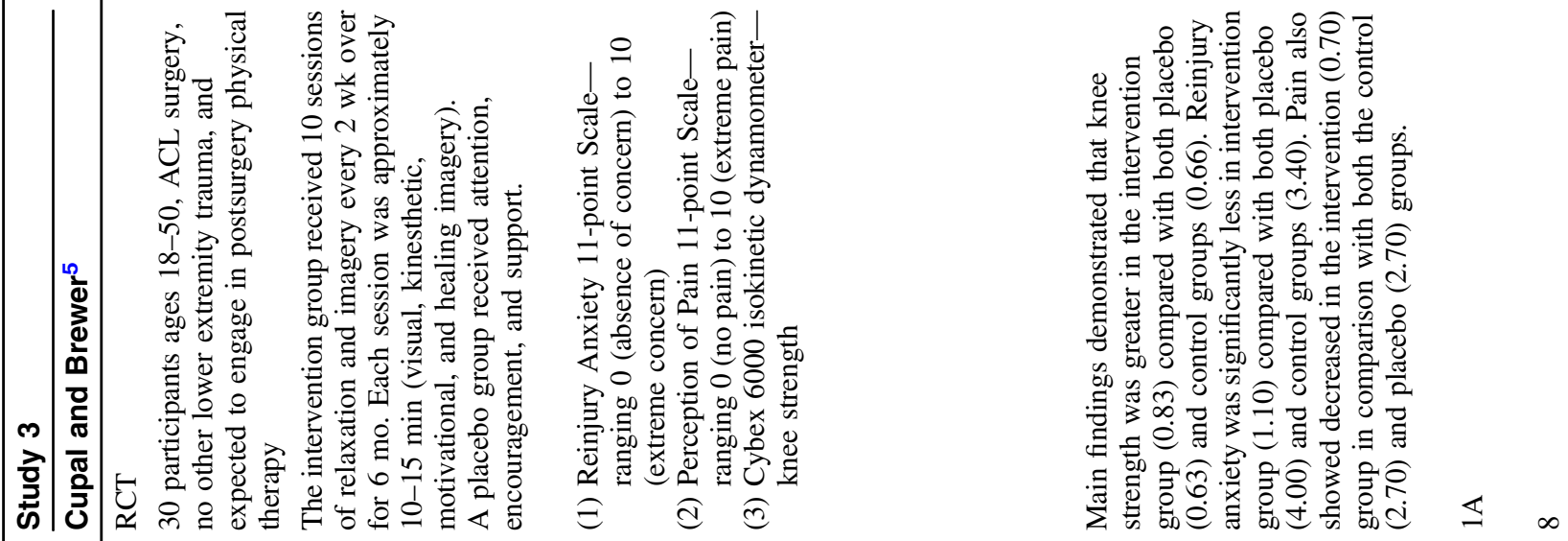

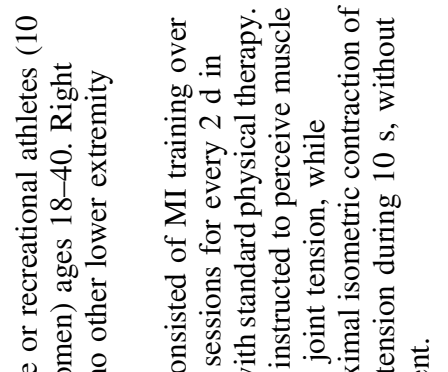

的

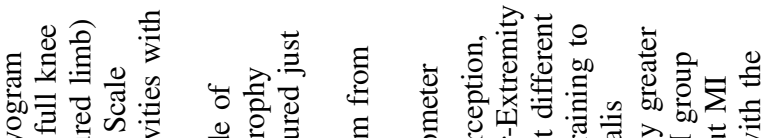

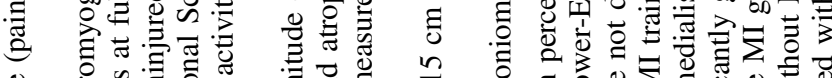



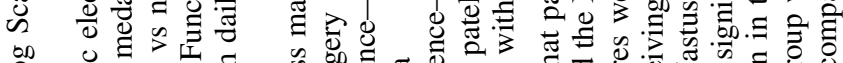

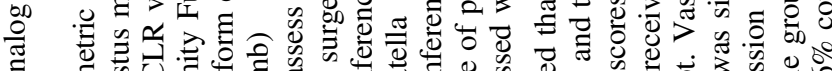

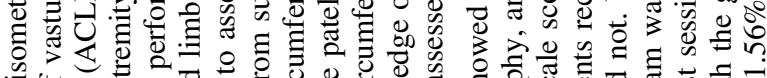



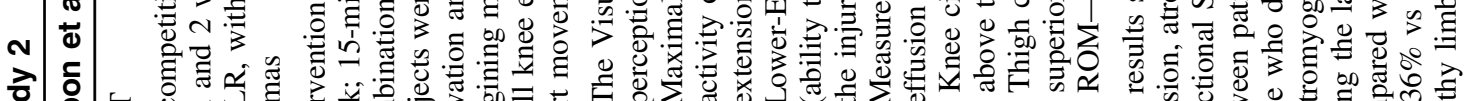

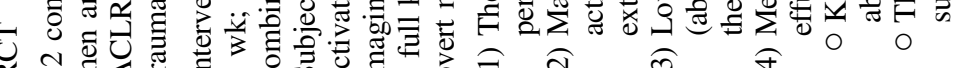

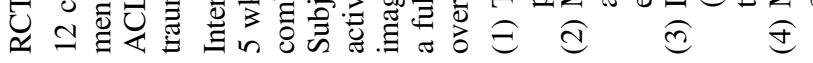

\section{1。 \\ 要过 \\ ริ ส \\ ॠ

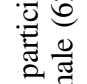 \\ 능}

$\stackrel{4}{3}$

$\stackrel{\Xi}{\Xi}$

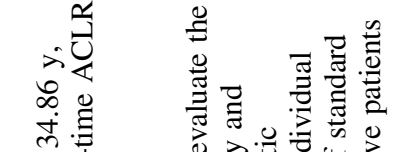

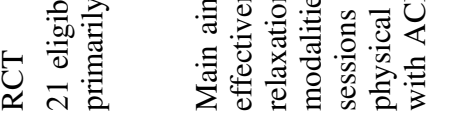

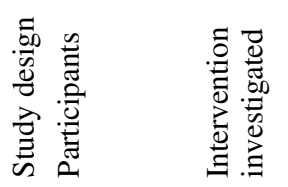

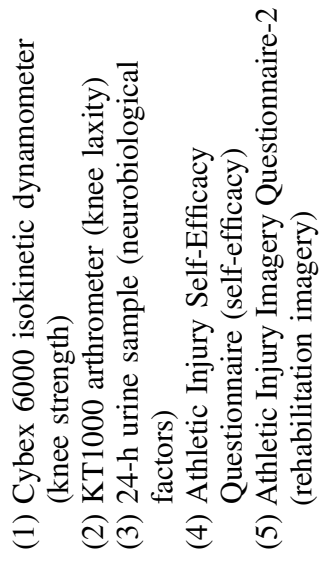

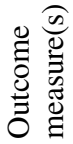

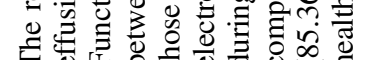

苛苛离害官

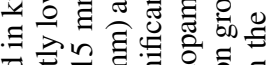

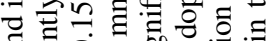

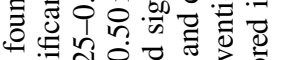

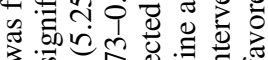

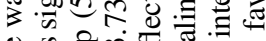

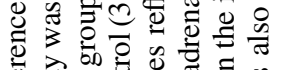

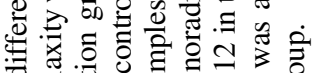

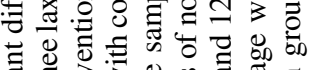

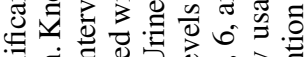

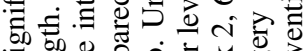

然券

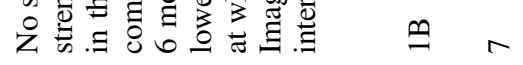

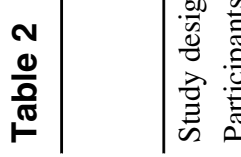

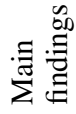

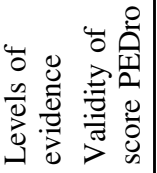




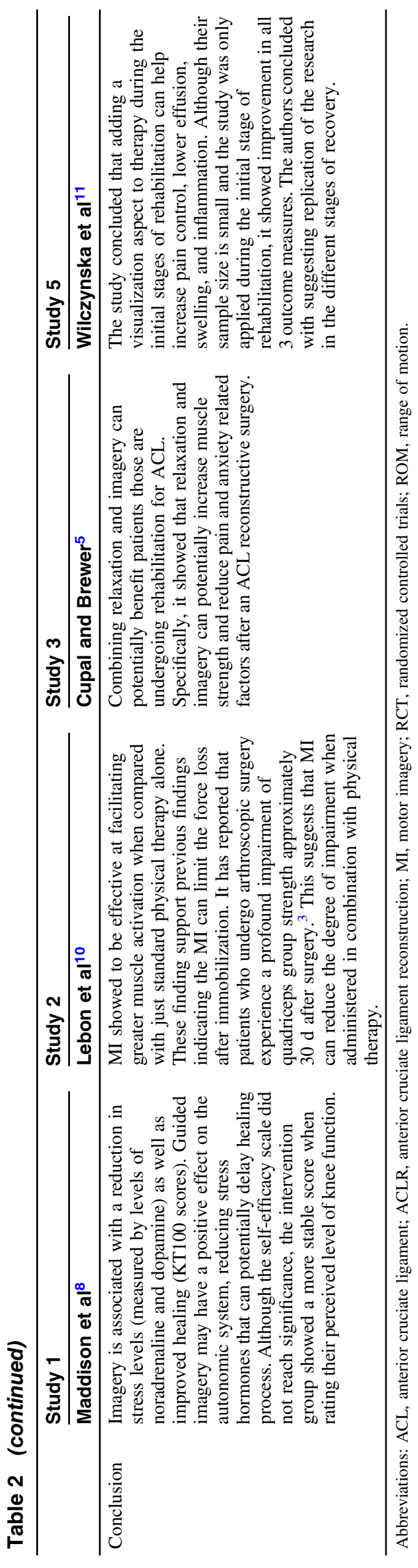


in the patient's ability to return to the same level of activity after surgery. ${ }^{5}$ Taking into consideration that patients report fear and confidence in their ability to reengage in competitive and recreational sports, implementing imagery techniques in physical therapy may help to alleviate these factors. ${ }^{9}$ The results of this critically appraised topic support the evidence that imagery can potentially reduce the fear of reinjury and pain in the ACLR population. Addressing the psychological component during the early stages of recovery has shown to be effective in reducing pain and increasing range of motion, while suggesting that the use of imagery is equally or more so important during the final stages of rehabilitation. ${ }^{11}$ Therefore, patients who report not being able to return to their preinjury activity or sport due to fear and pain can potentially benefit from imagery to ameliorate their distress. ${ }^{4}$ Imagery, in combination with physical therapy, can potentially promote greater activation of the quadriceps muscles and reduce ligamentous laxity, which can promote greater knee function. ${ }^{10}$ Reduction of neurobiological factors associated with anxiety and stress (noradrenaline and dopamine) is associated with utilizing imagery; this is significant due to the fact that stress hormones delay the healing process through pro-inflammatory cytokine activity. ${ }^{6}$ Considering the effectiveness of reinjury, pain questionnaires, and rating scales, clinicians should consider utilizing a tool (ie, Athletic Injury Self-Efficacy Questionnaire, 11-point pain/reinjury scale) to evaluate their patients during rehabilitation to identify those who would benefit from addressing psychological factors. Although the results support that imagery is an effective intervention, there are a couple of limitations to the selected studies. All 4 studies had a sample size of 30 or less, which is not necessarily a limitation, but there should be a focus on reproducing larger scale randomized controlled trials with a larger sample size to minimize the likelihood of reproducing falsepositive results. Also, more research involving longer follow-up time and single imagery modality should be explored. Finally, future research studies would benefit from including different study designs (cohort and prospective) in an effort to increase generalizability to the ACLR population. Another component to consider is the feasibility of implementing such an intervention in a clinical setting, addressing the barrier of insurance coverage as well as time allocation for the personnel leading the intervention. In conclusion, the results of the critically appraised topic support the effectiveness of imagery as a psychological intervention to reduce postpsychological consequences, while improving both functional and mental outcomes of patients with ACLR. Specifically, guided imagery has been shown to be associated with reduction of reinjury anxiety, lowered stress levels, increased muscle activation, and decreased pain perception. Therefore, ongoing research efforts are needed to examine the benefits of addressing psychological factors affecting injured athletes.

\section{References}

1. Arden CL, Webster KE, Taylor NF, Feller JA. Return to Sport following anterior cruciate ligament reconstruction surgery: a systematic review and meta-analysis of state of play. Br J Sports Med. 2011;45(7): 596-606. PubMed ID: 21398310 doi:10.1136/bjsm.2010.076364

2. van Grisven S, van Cingel RE, Holla CJ, van Loon CJ. Evidencebased rehabilitation following anterior cruciate ligament reconstruction. Knee Surg Sports Traumatol Arthrosc. 2010;18(8):1128-1144. PubMed ID: 20069277 doi:10.1007/s00167-009-1027-2

3. Beynnon BD, Johnson RJ, Abate JA, Fleming BC, Nichols CE. Treatment of anterior cruciate ligament injuries, part I. Am J Sports Med. 2005;33(10):1579-1602. PubMed ID: 16199611 doi:10.1177/ 0363546505279913

4. Medvecky MJ, Nelson S. Kinesiophobia and return to sports after anterior cruciate ligament reconstruction. J Connecticut Med. 2015; 79(3):155-157. PubMed ID: 26244221

5. Cupal DD, Brewer WD. Effects of relaxation and guided imagery on knee strength, reinjury anxiety, and pain following anterior cruciate ligament reconstruction. J Rehabil Psychology. 2001;46(1):28-43. doi:10.1037/0090-5550.46.1.28

6. Devan A, Magu NK, Siwach RC, Rohilla R, Sangwan SS. Functional outcome in athletes at five years of arthroscopic anterior cruciate ligament reconstruction. ISRN Orthop. 2011;2011:1-6. doi:10.5402/ 2011/570329

7. Schwab Reese LM, Pittsinger R, Yang J. Effectiveness of psychological intervention following sport injury. J Sport Health Sci. 2012; 1:71-79. doi:10.1016/j.jshs.2012.06.003

8. Maddison R, Prapavessis H, Clatworthy M, et al. Guided imagery to improve functional outcomes post-anterior cruciate ligament repair: Randomized-controlled pilot trial. Scand J Med Sci Sports. 2012; 22(6):816-821. PubMed ID: 21564307 doi:10.1111/j.1600-0838. 2011.01325.x

9. Tripp DA, Stanish W, Ebel-Lam A, Brewer BW, Birchard J. Fear of reinjury, negative affect, and catastrophizing predicting return to sport in recreational athletes with anterior cruciate ligament injuries at 1 year post surgery. J Rehabil Psychology. 2007;52(1):74-81. doi:10. 1037/0090-5550.52.1.74

10. Lebon F, Guillot A, Collet C. Increased muscle activation following motor imagery during the rehabilitation of the anterior cruciate ligament. Appl Psychophysiol Biofeedback. 2012;37:45-51. PubMed ID: 22127572 doi:10.1007/s10484-011-9175-9

11. Wilczynska D, Lysak A, Podczarska-Glowacka M. Imagery use in rehabilitation after the knee joint arthroscopy. Baltic J Health PA. 2015;7(4):93-101.

12. Ardern CL, Osterberg A, Tagesson S, Gauffin H, Webster KE, Kvist J. The impact of psychological readiness to return to sport and recreational activities after anterior cruciate ligament. Br J Sports Med. 2014;48: 1613-1619. PubMed ID: 25293342 doi:10.1136/bjsports-2014-093842 